\title{
Hábitos alimentarios de dos peces pelágicos Thunnus albacares y Acanthocybium solandri de la Reserva Marina de Galápagos
}

\author{
Feeding habits of two pelagic fish Thunnus albacares and Acanthocybium
} solandri from the Galapagos Marine Reserve

\section{Jorge Baque-Menoscal ${ }^{1}$, Diego Páez-Rosas ${ }^{2,3,4}$ y Matthias Wolff ${ }^{1,5}$}

\author{
'Departamento de Ciencias Marinas y Costeras, Fundación Charles Darwin, Av. Charles Darwin. Código Postal 200144, Isla \\ Santa Cruz, Galápagos, Ecuador \\ ${ }^{2}$ Programa PROMETEO, Secretaria Nacional de Educación Superior, Ciencia, Tecnología e Innovación del Ecuador, Calle \\ Whymper E7-37 y Alpallana, Quito, Ecuador. dpaez@usfq.edu.ec \\ ${ }^{3}$ Galapagos Science Center, Universidad San Francisco de Quito, Av. Alsacio Northia, Isla San Cristóbal, Galápagos, Ecuador \\ ${ }^{4}$ Laboratorio de Ecología de Pinnípedos 'Burney J. Le Boeuf', Centro Interdisciplinario de Ciencias Marinas, IPN, Av. \\ Instituto Politécnico Nacional s/n, Col. Playa Palo de Santa Rita, La Paz, B.C.S., México \\ ${ }^{5}$ Centro de Ecología Marina Tropical, Universidad de Bremen, Farenheitstrasse 6, Bremen, Alemania
}

\begin{abstract}
This study analyzed the trophic niche of two pelagic species that inhabit the Galapagos Marine Reserve, with the aim of understanding the interactions between these predators and other species. From June to October of 2009, 238 stomachs of Thunnus albacares and 151 of Acanthocybium solandri were analyzed from 4 specific sites of the Archipelago. Prey diversity reached an asymptotic level at 11 and 100 stomachs respectively. A total of 28 prey were found in T. albacores, in which the Humboldt squid Dosidicus gigas (36.68\%) was the numerically most represented, whereas 11 prey were found in $A$. solandri in which the flying fish Prognichthys tringa and D. gigas were the two main prey items representing $40 \%$ of the diet. In terms of weight and frequency of occurrence, the most important prey were the squids Histioteuthis heteropsis (8.75\%) and D. gigas (63.41\%) for T. albacares and Trachurus sp. (19.86\%) and Prognichthys tringa (1.19\%) for A. solandri. The index of relative importance (IIR) confirmed the importance of these items in the diet of both predators. The trophic breadth level classifies $T$. albacares as a specialist - and $A$. solandri as a generalist predator (Levin $=0.17$ and 0.65 , respectively). These results suggest important differences in feeding habits in the two apparently sympatric species, which allows them to minimize interactions between species and to maintain their sympatric presence in this region.
\end{abstract}

Key words: Trophic niche, sympatric, trophic similarity, Galapagos Islands

Resumen.- Este estudio analiza el nicho trófico de dos especies pelágicas presentes en la Reserva Marina de Galápagos, con la finalidad de comprender las interacciones que estos depredadores tiene con otras especies. De junio a octubre del 2009 se analizaron 238 estómagos de Thunnus albacares y 151 de Acanthocybium solandri provenientes de 4 sitios específicos del Archipiélago. La diversidad de presas alcanzó el nivel asintótico con 11 y 100 estómagos respectivamente. Un total de 28 presas fueron encontradas en T. albacares, de las cuales el calamar gigante Dosidicus gigas $(36,68 \%)$ fue la presa numéricamente más representativa, mientras que 11 presas fueron encontraron en $A$. solandri, de las cuales el pez volador Prognichthys tringa y el calamar gigante $D$. gigas fueron las presas principales representando el $40 \%$ de la dieta. En términos gravimétricos y porcentajes de frecuencia las presas más importantes para T. albacares fueron los calamares Histioteuthis heteropsis $(8,75 \%)$ y D. gigas $(63,41 \%)$, mientras que para $A$. solandri fueron los peces Trachurus sp. $(19,86 \%)$ y Prognichthys tringa $(1,19 \%)$. El índice de la importancia relativa (IIR) confirmó la importancia de estas presas en la dieta de ambos depredadores. El nivel de amplitud trófica mostró a $T$. albacares como un depredador especialista; mientras que $A$. solandri resultó ser un depredador generalista (Levin $=0,17$ y 0,65 , respectivamente). Estos resultados muestran diferencias en los hábitos alimentarios de especies aparentemente simpátricas, adaptación que les permitiría disminuir los niveles de interacción entre ellas y así facilitar su permanencia en esta región.

Palabras clave: Nicho trófico, simpatría, similitud trófica, Islas Galápagos 


\section{INTRODUCCIÓN}

Los estudios tróficos en depredadores marinos, aportan de manera integral información biológica básica sobre el comportamiento ecológico de estas especies lo cual permite comprender las diversas interacciones que se establecen entre estas poblaciones, sus presas y sus competidores. Sin embargo la gran mayoría de estudios en los grandes peces pelágicos se han enfocado en poblaciones aisladas, sin considerar la asociación entre varias especies.

Las asociaciones poliespecíficas incluyen a diferentes especies que viajan, interactúan y se alimentan juntas por distintos periodos de tiempo. Las interacciones tróficas inter-específicas involucradas, no están bien comprendidas, a pesar de diversas observaciones que se han hecho en el mar (Au 1991). Se cree que uno de los principales motivos para la formación de estas asociaciones es la búsqueda de alimento. Frecuentemente están involucradas varias especies de importancia comercial de diferentes tamaños y hábitos, que se desplazan en zonas tropicales y en ambientes pelágicos (Arenas et al. 1999).

El atún aleta amarilla Thunnus albacares (Bonaterre, 1788) y el guajo Acanthocybium solandri (Cuvier, 1832) son especies pelágicas, que habitan las zonas tropicales y subtropicales; distribuidas en el Océano PacíficoOriental, desde el sur de California EUA, hasta Perú (Alverson 1963, Fisher et al. 1995, Theisen et al. 2008). Ambos peces se encuentran en la zona epipelágica y rara vez sobrepasan los $200 \mathrm{~m}$ de profundidad. El guajo además prefiere zonas cercanas a arrecifes y paredes rocosas de islas oceánicas (Eslava et al. 2003, Oxenford et al. 2003, Sepúlveda et al. 2011). Ambas especies son muy activas y pueden atravesar grandes distancias en corto tiempo, por lo que requieren de una alta cantidad de energía (Olson \& Boggs 1986, Oxenford et al. 2003). Por lo cual diversos autores consideran que la disponibilidad de alimento es un factor determinante en la abundancia y distribución de estos escómbridos.

Frecuentemente estos peces pelágicos están asociados a otras especies, como estrategia para reducir la presión de depredación o incrementar el éxito en la alimentación (Nikolsky 1963, Au 1991). En zonas como el Pacífico ecuatorial se conoce muy poco con respecto a sus hábitos alimentarios. Sin embargo, estudios realizados en el norte del Océano Pacífico Oriental y el Golfo de México, se determinó que éstos peces consumen varias especies de peces, cefalópodos y crustáceos, de las que se destacan las presas Sardinops sagax, Clupea harengus, Auxis spp., Dosidicus gigas y Pleuroncodes planipes (Alverson 1963, Galván-Magaña 1988, Oxenford et al.2003).

Ambas especies se encuentran asociadas en aguas de la Reserva Marina de Galápagos (RMG), la cual es una zona con características subtropicales influenciadas por las diferentes corrientes marinas que circundan durante el año (Banks 2002). Una de las corrientes que más nutrientes trae al archipiélago es la Corriente submarina Ecuatorial (Corriente de Cromwell), que produce zonas de afloramiento continuo en las costas occidentales del archipiélago durante todo el año (Palacios 2002, Palacios et al. 2006). La productividad anual promedio refleja valores altos en las islas del oeste (Isabela y Fernandina) y una productividad promedio en el resto del archipiélago; sin embargo se debe destacar que existen zonas cercanas a las islas del sureste del archipiélago (San Cristóbal, Santa Fe y Santa Cruz) que presentan una producción más alta de la esperada (Banks 2002), lo cual brinda condiciones óptimas para la presencia de estos grandes peces pelágicos alrededor del archipiélago.

Actualmente, la pesquería de ambas especies tiene gran importancia para los pescadores artesanales de las Islas Galápagos (Castrejón 2008), sin embargo no existen estudios sobre su biología básica, a pesar de su gran importancia ecológica dentro de la trama trófica de la región. El objetivo de esta investigación consistió en determinar el nicho trófico de las poblaciones de Thunnus albacares y Acanthocybium solandri, presentes en la Reserva Marina de las Islas Galápagos; con la finalidad de determinar el rol trófico de estas especies en la región. El estudio incluyó un análisis cuantitativo (numérico, gravimétrico, porcentaje de frecuencia) de las dietas y cálculos de: diversidad, amplitud y similitud trófica de ambas especies.

\section{Materiales y MÉTODOS}

Las muestras provienen de un tipo de pesca artesanal (línea con anzuelo) efectuado diariamente por los pescadores de la Isla Santa Cruz, Galápagos $\left(0^{\circ} 67^{\prime} \mathrm{S}\right.$, $\left.90^{\circ} 33^{\prime} \mathrm{O}\right)$, quienes dirigen sus faenas a cuatro puntos específicos dentro de la Reserva Marina de Galápagos (Banco Ruso, San Luis, Cuatro Hermanos, La Viuda) (Fig. 1). Se realizaron 2 muestreos semanales en el muelle de desembarque de Puerto Ayora entre junio y octubre de 2009. De cada pez se obtuvieron medidas morfométricas (peso del cuerpo, longitud furcal recta) y se extrajo el 


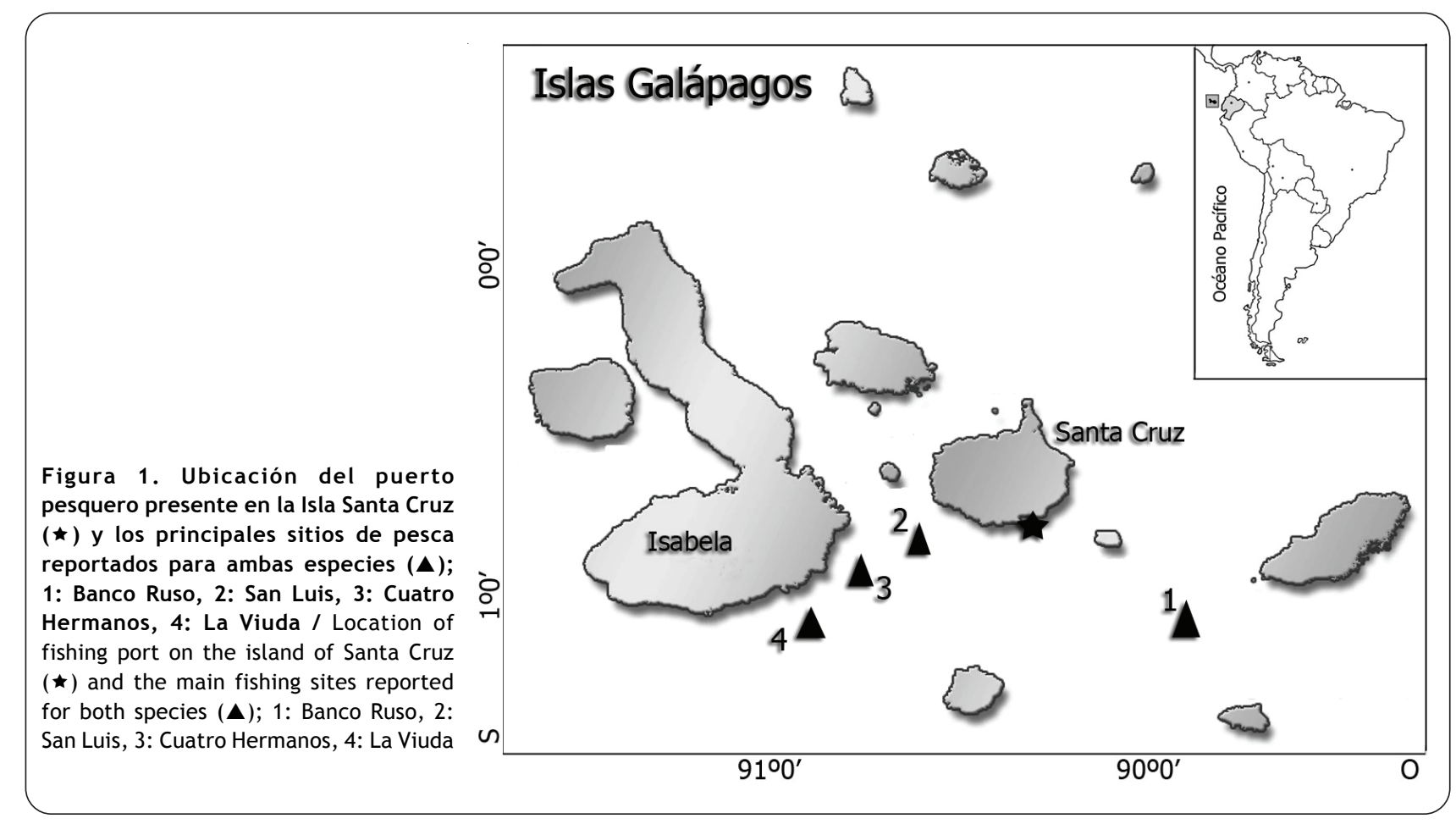

estómago, logrando recolectar un total de 238 estómagos de Thunnus albacares y 151 de Acanthocybium solandri.

Los estómagos fueron guardados en bolsas plásticas de cierre hermético, etiquetadas con fecha y sitio de recolecta, para luego ser llevados al laboratorio en estado fresco. Se calculó el porcentaje de llenado (repleción gástrica) y el grado de digestión de las presas encontradas en cada estómago de acuerdo a la escala propuesta por Galván et al. (1989): estado de digestión 1 (presas recientes): presas completas, estado 2 (intermedio): presa en descomposición pero con presencia de músculo u otros tejidos, estado 3 (semi digerido): presencia de esqueletos de peces o exoesqueletos de crustáceos, y estado 4 (digerido): presencia de partes duras, otolitos de peces y mandíbulas de cefalópodos. Posteriormente se procedió a pesar el contenido estomacal y separar las presas para su identificación.

Las piezas esqueléticas recuperadas, se colocaron en frascos con alcohol al 50\%, para finalmente proceder a identificarlas al nivel taxonómico más preciso posible, con ayuda de una lupa estereoscópica, basándose en literatura especializada. En el caso de las mandíbulas de cefalópodos y restos de crustáceos se utilizaron claves publicadas (Garth \& Stephenson 1966, Wolff 1984, Clarke 1986), mientras que la determinación taxonómica de los peces fue hecha en base a las características del esqueleto axial y la morfometría de sus otolitos. Para el conteo de vértebras se utilizó el criterio propuesto por Clothier \& Baxter (1969) y para los otolitos se utilizó información bibliográfica de la zona (García-Godos 2001, Páez-Rosas 2008, Salazar-Aldáz 2008).

Con la finalidad de disminuir la variabilidad al momento de analizar y comparar los datos de ambas especies, únicamente se tomaron en cuenta los estómagos de ejemplares adultos, con base en la longitud corporal promedio de madurez sexual reportada para ambas especies; en el caso del atún aleta amarilla fueron considerados los individuos que sobrepasan los $85 \mathrm{~cm}$ de longitud furcal recta (LF) (Shaefer 1998); mientras que para el guajo se consideraron a los individuos mayores de $105 \mathrm{~cm} \mathrm{LF}$ (Oxenford et al. 2003).

Para estimar la representatividad del tamaño de muestra se aplicó la rutina propuesta por Ferry et al. (1997) y aplicada por Páez-Rosas \& Aurioles-Gamboa (2010) para estudios de hábitos alimentarios en depredadores de la zona. Este método consiste en calcular la media acumulada y la desviación estándar de un grupo de curvas de diversidad generadas con los datos de abundancia de las presas, mediante el Índice de Shannon-Wiener 'H' (Krebs 1999). Las curvas de diversidad se derivan de una rutina 
diseñada en el programa MATLAB, que computa 500 permutaciones al azar con todos los datos originales, manteniendo un margen de error de 0,05. Este error se obtiene mediante el coeficiente de variación de los datos y muestra cuando la media acumulada llega a una asíntota que permite determinar el tamaño de muestra apropiado para caracterizar la dieta del depredador.

En el análisis cuantitativo de los componentes alimentarios se utilizaron los métodos numérico $(\mathrm{N})$, gravimétrico (G) y frecuencia de ocurrencia (FO) propuestos por Hyslop (1980) y Caillet et al. (1986). Asimismo, el aporte de cada presa a la dieta del depredador se cuantificó usando el Índice de Importancia Relativa (IIR) propuesto por Pinkas et al. (1971) y modificado por Stevens et al. (1982); quienes sugieren el siguiente algoritmo:

$$
I I R=(N+G) F O
$$

donde $\mathrm{N}$ es la contribución numérica porcentual de la presa; $\mathrm{G}$ es la proporción del peso total de cada presa con respecto al peso total de alimento; mientras que FO es la frecuencia de ocurrencia porcentual de cada presa. Este método es utilizado con la finalidad de aportar información que equilibre los resultados obtenidos por los métodos anteriores, los cuales por separado pueden subestimar o sobrestimar la importancia de ciertas presas (Stevens et al. 1982).

Para determinar si ambas especies presentaron un grado de especialización sobre los recursos alimenticios utilizamos el Î́ndice Estandarizado de Levin ' $\mathrm{B}_{i}$ ' (Krebs 1999). Ese índice mide la amplitud trófica de una especie otorgándole la condición de especialista o generalista, con base en el siguiente algoritmo:

$$
B_{i}=\frac{1}{n-1\left\{\left(1 / \sum J P_{i j}{ }^{2}\right)-1\right\}}
$$

donde $\mathrm{JP}_{\mathrm{ij}}$ es el porcentaje del Índice de importancia de presa (IIR) de la presa (i) en la dieta de la especie (j); n es el número total de especies presas presentes en la dieta. Los valores de este índice fluctúan de 0 a 1 , por debajo de 0,6 indican una dieta dominada por pocas presas, por lo tanto se trata de un depredador especialista y valores mayores a 0,6 revelan dietas de depredadores generalistas (Krebs 1999).
El grado de similitud trófica, se midió utilizando un análisis de similitud (ANOSIM), el cual es considerado como análogo al análisis de varianza (ANDEVA). Esta prueba denominada como de permutación aleatoria se realizó con ayuda del programa PRIMER 6, cuyos resultados permiten determinar si existen diferencias significativas en la composición de la dieta de los consumidores. Clarke \& Warwick (2001) plantean la utilización de una hipótesis nula (Ho) en el análisis de similitud; esto con base en el estadístico de prueba R, el cual se obtiene del siguiente algoritmo:

$$
R=\frac{\left(r_{B}-r_{W}\right)}{12 M}
$$

donde $r_{B}$ es el promedio de los intervalos de similitud de todos los pares de réplicas entre los diferentes sectores; $r_{w}$ es el promedio de todos los intervalos de similitud dentro de los sectores; mientras que $\mathrm{M}$ es el resultado de la siguiente ecuación: $\mathrm{M}=\mathrm{n}(\mathrm{n}-1) / 2$; donde $\mathrm{n}$ es el número total de muestras en consideración. Los valores de $\mathrm{R}$ varían de 0 a 1 indicando un grado de discriminación entre muestras, el cual depende de la riqueza y abundancia de especies presas en cada organismo (Clarke \& Warwick 2001); valores de 0 indican similitud entre y dentro de los grupos, mientras que valores cercanos a 1 sugieren dietas diferentes.

\section{Resultados}

\section{COMPOSICIÓN DE LA DIETA}

Las curvas de diversidad de presas en Thunnus albacares alcanzaron la asíntota en 11 estómagos; mientras que en Acanthocybium solandri fue en 100 estómagos; lo cual refleja que el tamaño de muestra utilizado en este estudio (45 y 118 estómagos respectivamente) es el adecuado para describir con precisión la composición de la dieta de ambas especies en la zona de estudio (Fig. 2).

Del total de estómagos de Thunnus albacares analizados, 71 estuvieron vacíos y 167 presentaron contenido estomacal, mientras que para Acanthocybium solandri 57 estómagos estuvieron vacíos y 94 tenían contenido. De forma general para ambas especies el 50,90\% de las presas estaban digeridas (estado 4 de digestión); el 6,49 \% en estado 3; el 15,34\% estaban en el estado 2 y el $27,25 \%$ en estado 1 . 
Para definir la dieta y las posibles diferencias en el nicho trófico de ambas poblaciones únicamente se analizaron los estómagos de organismos adultos, clasificando la muestra en 45 estómagos de Thunnus albacares y 118 estómagos de Acanthocybium solandri (Fig. 3).

El espectro trófico de Thunnus albacares estuvo integrado por 28 presas, de las cuales 16 fueron peces, 11 cefalópodos y 1 crustáceo; lográndose identificar 19 a nivel de especie, 6 a nivel de género, 2 a nivel de familia y 1 a nivel de orden (Tabla 1). El espectro trófico de Acanthocybium solandri estuvo compuesto de 11 presas: 7 peces, 3 cefalópodos y 1 crustáceo; lográndose identificar 7 a nivel de especie, 3 a nivel de género y 1 a nivel de orden (Tabla 2).
Figura 2. Curvas de diversidad de presas (media acumulada \pm desviación estándar) presentes en la dieta de las poblaciones de atún aleta amarilla (Thunnus albacares) y guajo (Acanthocybium solandri), basadas en muestras de contenido estomacal de ambas especies. El valor del índice acumulado de diversidad fue en base al Índice de Shannon-Wiener $\left(\mathrm{H}^{\prime}\right)$. ( $\mathrm{n}=$ tamaño muestral óptimo para cada especie) / Mean cumulative prey diversity curves and SD for populations of yellowfin tuna (Thunnus albacares) and wahoo (Acanthocybium solandri), based on samples of the stomach contents of each species. Cumulative prey diversity was based on the Shannon-Wiener Index $\left(\mathrm{H}^{\prime}\right)$. ( $\mathrm{n}=$ optimum sample size for each species)

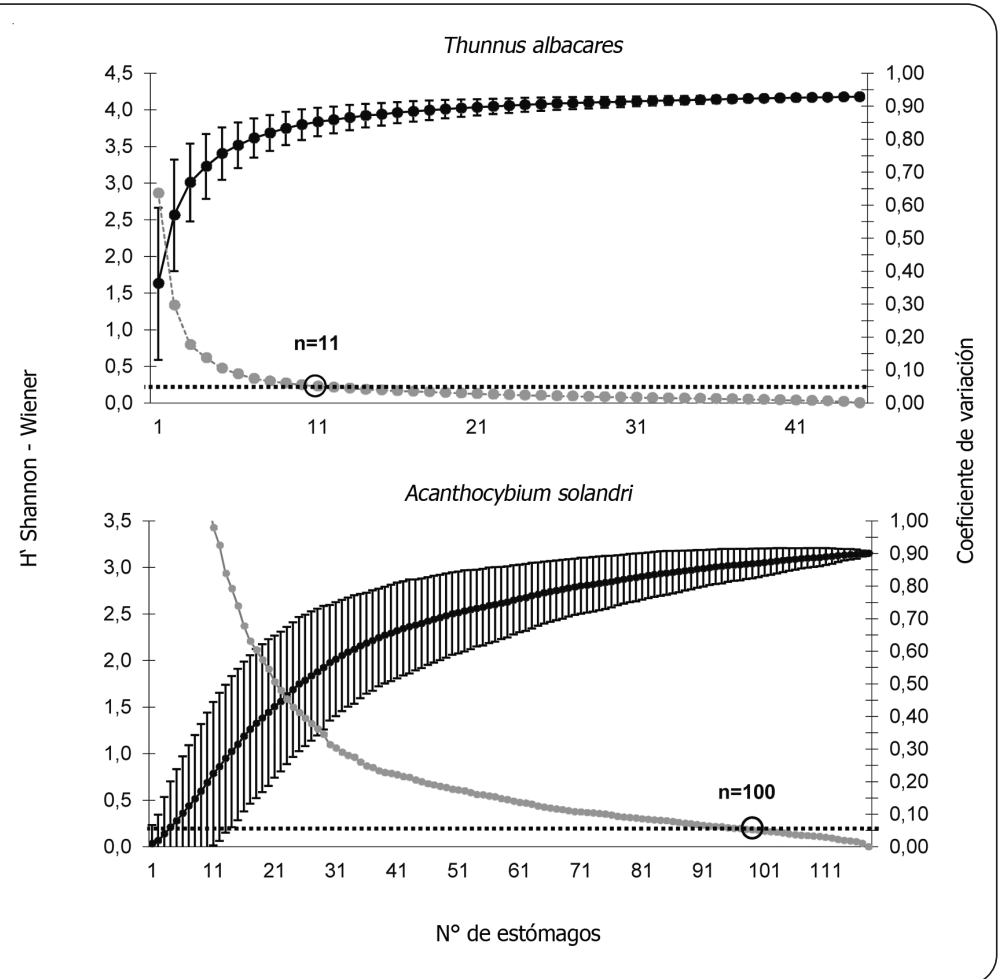

Figura 3. Estructura de tallas $(\mathrm{cm})$ de las muestras de atún aleta amarilla (Thunnus albacares) y guajo (Acanthocybium solandri) capturadas en la Reserva Marina de Galápagos / Size structure $(\mathrm{cm})$ of the samples of yellowfin tuna (Thunnus albacares) and wahoo (Acanthocybium solandri) collected in the Galapagos Marine Reserve

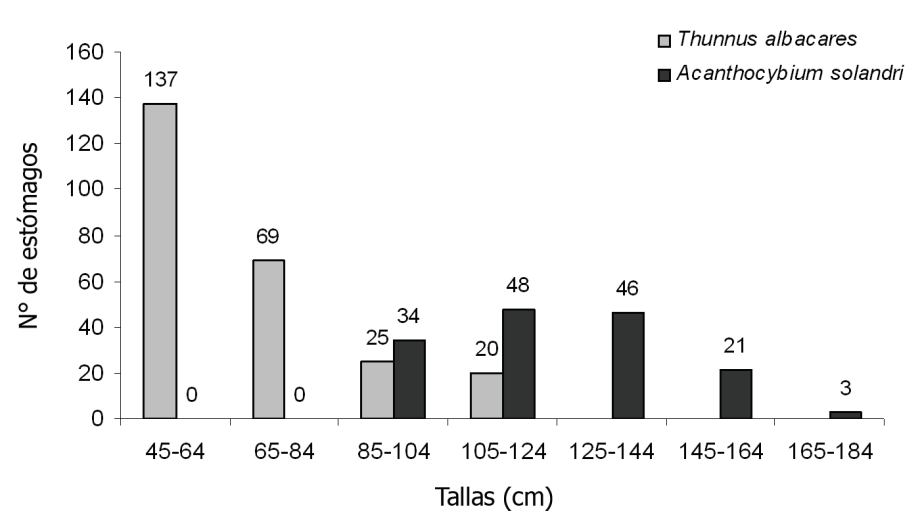

Vol. 47, Nº1, 2012 
Tabla 1. Espectro trófico del atún aleta amarilla (Thunnus albacares) presente en la Reserva Marina de Galápagos. Expresado en valores porcentuales de los métodos numérico $(\mathrm{N})$, gravimétrico $(\mathrm{G})$, frecuencia de ocurrencia $(\mathrm{FO})$ e índice de importancia relativa (IIR) / Trophic spectrum of yellowfin tuna (Thunnus albacares) present in the Galapagos Marine Reserve, expressed in percentage values of numerical methods $(\mathrm{N})$, gravimetric $(\mathrm{G})$, occurrence frequency (FO) and relative importance index (IIR)

\begin{tabular}{|c|c|c|c|c|}
\hline Especies presa & $\% \mathrm{~N}$ & $\% \mathrm{G}$ & $\% \mathrm{FO}$ & IIR \\
\hline \multicolumn{5}{|l|}{ Cefalópodos } \\
\hline Abroliopsis affinis & 1,41 & 0,67 & 9,75 & 9,34 \\
\hline Alloposus mollis & 1,56 & 1,95 & 17,07 & 52,34 \\
\hline Argonauta sp. & 2,66 & 2,36 & 21,95 & 138,11 \\
\hline Dosidicus gigas & 36,67 & 2,64 & 63,41 & 2493,73 \\
\hline Histioteuthis heteropsis & 1,25 & 8,75 & 9,75 & 107,06 \\
\hline Mastigoteuthis dentata & 0,62 & 1,93 & 7,31 & 8,86 \\
\hline Octopus rubescens & 7,68 & 1,75 & 24,39 & 229,99 \\
\hline Onychoteuthis banksii & 11,44 & 2,09 & 53,65 & 1283,73 \\
\hline Pterygioteuthis giardi & 1,25 & 1,17 & 9,75 & 14,33 \\
\hline Sthenoteuthis oualaniensis & 1,09 & 5,79 & 14,63 & 92,97 \\
\hline Thysanoteuthis rhombus & 0,15 & 0,26 & 2,43 & 0,10 \\
\hline Subtotal & 65,83 & 29,39 & 234,14 & 8191,97 \\
\hline \multicolumn{5}{|l|}{ Peces } \\
\hline Chromis atrilobata & 0,78 & 5,47 & 4,87 & 20,94 \\
\hline Diplectrum sp. & 0,47 & 3,63 & 4,87 & 8,33 \\
\hline Epinephelus sp. & 1,25 & 5,44 & 12,19 & 83,29 \\
\hline Exocoetus monocirrhus & 1,88 & 3,33 & 19,51 & 122,27 \\
\hline Hemiramphus saltador & 0,15 & 7,39 & 2,43 & 2,82 \\
\hline Isacia sp. & 0,15 & 4,12 & 2,43 & 1,57 \\
\hline Fam. Malacantidae & 0,62 & 3,66 & 4,87 & 11,21 \\
\hline Oxyporhamphus micropterus & 3,60 & 5,02 & 31,70 & 574,11 \\
\hline Fam. Myctophidae & 4,54 & 1,01 & 12,19 & 56,21 \\
\hline Myctophum nitidulum & 0,15 & 6,14 & 2,43 & 2,35 \\
\hline Opisthonema berlangai & 3,29 & 6,04 & 14,63 & 291,08 \\
\hline Paralabrax sp. & 0,15 & 5,24 & 2,43 & 2,01 \\
\hline Prognichthys tringa & 5,64 & 3,46 & 48,78 & 953,57 \\
\hline Selar crumenopthalmus & 0,15 & 0,28 & 2,43 & 0,11 \\
\hline Trachurus sp. & 0,15 & 3,18 & 2,43 & 1,21 \\
\hline Vinciguerria lucetia & 2,03 & 4,01 & 9,75 & 79,90 \\
\hline Subtotal & 25,07 & 67,51 & 178,04 & 2211,04 \\
\hline \multicolumn{5}{|l|}{ Crustaceos } \\
\hline Megalopa de cangrejo & 9,09 & 3,09 & 12,19 & 342,73 \\
\hline Subtotal & 9,09 & 3,09 & 12,19 & 342,73 \\
\hline
\end{tabular}

El método numérico determinó que la presa más representativa para el atún aleta amarilla fue el calamar gigante Dosidicus gigas (36,68\%), seguido del calamar Onychoteuthis banksii $(11,44 \%)$; mientras que para el guajo las presas más representativas fueron el pez volador Prognichthys tringa (20\%) y el calamar gigante Dosidicus gigas $(20 \%)$ (Tablas 1 y 2). Gravimétricamente, las presas de mayor importancia para el atún aleta amarilla fueron el calamar Histioteuthis heteropsis (8,75\%), seguido del pez Hemiramphus saltador (7,4\%); en el caso del guajo las presas mas importantes fueron el pez Trachurus sp. $(19,86 \%)$, seguido de Sphyraena idiastes (18,33\%) (Tablas 1 у 2$)$.

De acuerdo con el índice de importancia relativa (IIR) los cuatro componentes principales en la dieta del atún
Tabla 2. Espectro trófico del guajo (Acanthocybium solandri) presente en la en la Reserva Marina de Galápagos. Expresado en valores porcentuales de los métodos numérico $(\mathrm{N})$, gravimétrico (G), frecuencia de ocurrencia (FO) e índice de importancia relativa (IIR) / Trophic spectrum of wahoo (Acanthocybium solandri) present in the Galapagos Marine Reserve. Expressed in percentage values of numerical methods $(\mathrm{N})$, gravimetric $(\mathrm{G})$, occurrence frequency $(\mathrm{FO})$ and relative importance index (IIR)

\begin{tabular}{lrrrr}
\hline \multicolumn{1}{c}{ Especies presa } & $\% \mathrm{~N}$ & $\% \mathrm{G}$ & $\% \mathrm{FO}$ & $\mathrm{IIR}$ \\
\hline Cefalópodos & & & & \\
$\quad$ Dosidicus gigas & 20,000 & 7,110 & 0,955 & 25,881 \\
$\quad$ Onychoteuthis banksii & 4,000 & 7,721 & 0,239 & 2,797 \\
$\quad$ Sthenoteuthis oualaniensis & 8,000 & 6,117 & 0,477 & 6,738 \\
$\quad$ Subtotal & 32,000 & 20,948 & 1,671 & 35,417 \\
& & & & \\
Peces & 4,000 & 2,732 & 0,239 & 1,607 \\
Diplectrum sp. & 8,000 & 3,985 & 0,477 & 5,721 \\
Exocoetus monocirrhus & 4,000 & 2,587 & 0,239 & 1,572 \\
Ophistonema berlangai & 4,000 & 5,361 & 0,239 & 2,234 \\
Paralabrax sp. & 20,000 & 7,317 & 1,193 & 32,598 \\
Prognichthys tringa & 4,000 & 19,333 & 0,239 & 5,569 \\
Sphyraena idiastes & 8,000 & 19,861 & 0,477 & 13,299 \\
Trachurus sp. & 52,000 & 61,178 & 3,103 & 62,600 \\
$\quad$ Subtotal & & & & \\
$\quad$ Crustáceos & 16,000 & 17,874 & 0,239 & 8,085 \\
Megalopa de cangrejo & 16,000 & 17,874 & 0,239 & 8,085 \\
$\quad$ Subtotal & & & &
\end{tabular}

aleta amarilla fueron: Dosidicus gigas (IIR=57,30\%), Onychoteuthis banksii $(\mathrm{IIR}=11,95 \%)$, Prognichthys tringa $(\mathrm{IIR}=8,87 \%)$ y Oxyporhamphus micropterus micropterus $(\mathrm{IIR}=5,34 \%)$ (Fig. 4, Tabla 1). Mientras que los tres componentes principales en la dieta del guajo fueron: Prognichthys tringa (IIR=30,72\%), Dosidicus gigas $(\mathrm{IIR}=24,39 \%)$ y Trachurus $\mathrm{sp}$. (IIR=12,53\%) (Fig. 4 , Tabla 2).

\section{DiVERSIDAD DE PRESAS Y AMPLITUD DEL NICHO TRÓFICO}

La heterogeneidad presente en la dieta de ambas especies fue calculada con base en la diversidad promedio de presas presente en la dieta; en el caso de Thunnus albacares esta fue considerablemente mayor $\left(\mathrm{H}^{\prime}=2,7 \pm\right.$ 0,3 ) en comparación a la observada en la dieta de Acanthocybium solandri $\left(\mathrm{H}^{\prime}=1,2 \pm 0,4\right)$. La dieta de Thunnus albacares muestra que esta especie se alimenta preferentemente de presas de hábitos mesopelágicos (76,8\%), pero también de especies epipelágicas $(18,1 \%)$ (Fig. 5). Se observa una situación inversa con respecto a Acanthocybium solandri, el cual registró una dieta dominada por especies epipelágicas $(55,4 \%)$ seguida por especies mesopelágicas $(33,4)$ (Fig. 5).

Los valores de amplitud trófica calculados para Thunnus albacares en el área de estudio lo muestran 
Figura 4. Porcentaje de Índice de Importancia Relativa (IIR) de las principales presas identificadas para las poblaciones de atún aleta amarilla (Thunnus albacares) y guajo (Acanthocybium solandri) encontrados en la Reserva Marina de Galápagos / Relative importance index (RII) of the main prey observed in the diet of the populations of yellowfin tuna (Thunnus albacares) and wahoo (Acanthocybium solandri) found in the Galapagos Marine Reserve

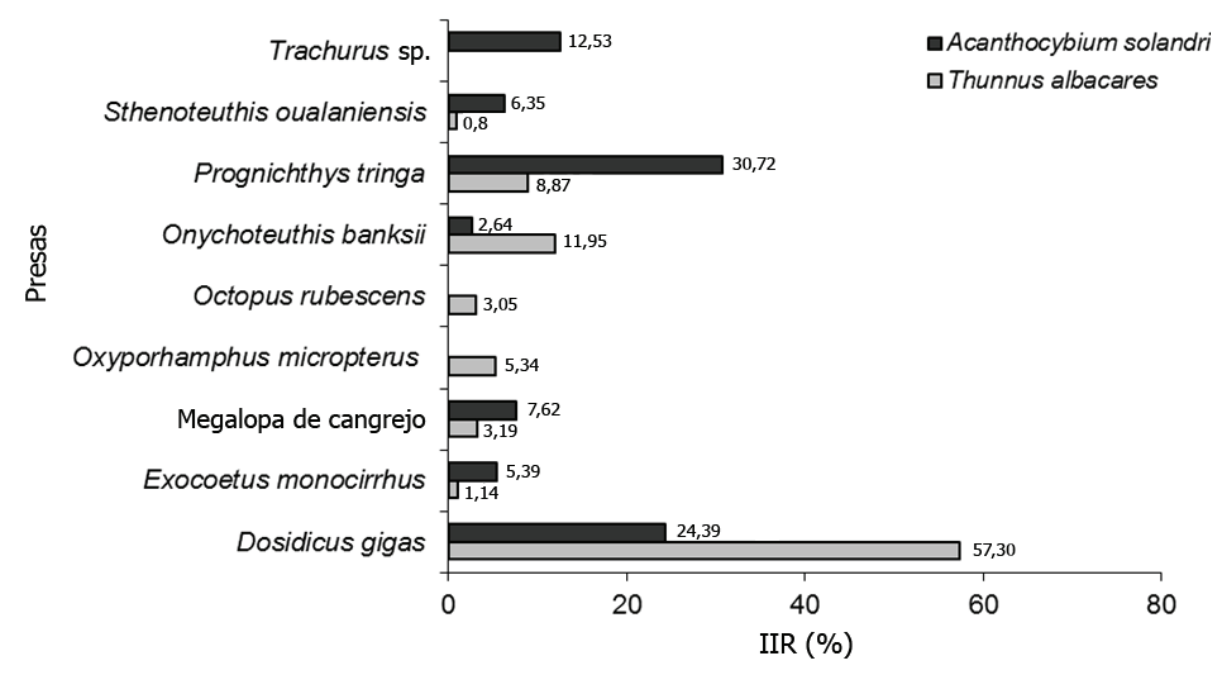

Figura 5. Importancia relativa del tipo de hábitat de las presas en la dieta de Thunnus albacares y Acanthocybium solandri basados en los valores de IIR / Relative importance of the prey habitat type in the diet of Thunnus albacares and Acanthocybium solandri based on the IIR values

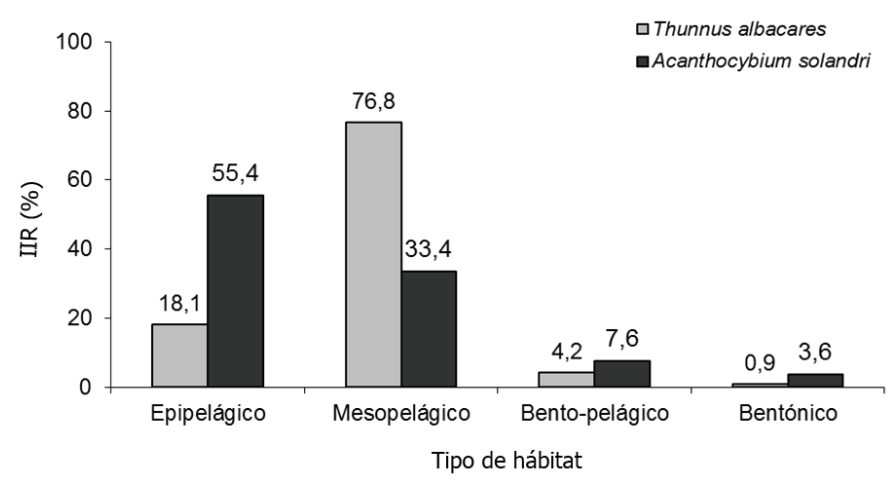

como un depredador de tipo especialista $B_{i}=0,17$ ya que a pesar de presentar un amplio espectro alimentario, este depredador dirige la mayor parte de su alimentación a un grupo limitado de especies. Mientras que Acanthocybium solandri resultó ser una especie con hábitos generalistas $B_{i}=0,65$; debido a que utiliza sus recursos de una forma más homogénea.

\section{Similitud TRÓfiCA ENTRE AMBAS ESPECIES}

El análisis de similitud (ANOSIM) mostró una alta similitud en sus hábitos alimentarios de Thunnus albacares y Acanthocybium solandri $(\mathrm{R}=0,134 ; P=0,05)$. Esto debido a que el calamar gigante Dosidicus gigas fue una de las presas que consumieron en mayor proporción tanto Thunnus albacares $(\mathrm{IIR}=57,30 \%)$ como Acanthocybium solandri $(\mathrm{IIR}=24,39 \%)$ (Fig. 4$)$.

\section{Discusión}

El estudio de la dinámica de las interacciones tróficas de los depredadores marinos ha adquirido gran relevancia en los últimos años; ya que provee información biológica importante para entender las relaciones intra e interespecíficas de las especies, situación que se vuelve valiosa al momento de estudiar especies de interés comercial (Wooton 1990).

La dieta de Thunnus albacares determinada en el presente estudio coincide con estudios recientes realizados en otras zonas del Océano Pacífico Oriental como la región de California y Baja California (GalvánMagaña 1988, Román-Reyes 2005, Alatorre-Ramírez 2007.), donde el calamar gigante Dosidicus gigas es una de sus principales presas. Sin embargo, difiere con lo reportado por Alverson (1963), quien observó que la dieta de las 
poblaciones de Thunnus albacares presentes en el Pacífico Ecuatorial consistía principalmente por peces y pequeños crustáceos.

En términos de diversidad de la dieta los resultados de este estudio muestran diferencias entre el espectro trófico de las poblaciones de Thunnus albacares presentes en la Reserva Marina de Galápagos ( 28 presas) y el observado en otras regiones del Océano Pacífico Oriental. Galván-Magaña (1988) y Román-Reyes (2005) reportaron un espectro trófico más amplio en la región de Baja California ( $\sim 55$ presas); lo cual podría deberse a las diferencias en la disponibilidad de presas presente en ambas regiones. Se conoce que existe una mayor diversidad íctica en el Golfo de California (908 especies) (Hastings et al. 2010), en comparación a la reportada en la región de las Islas Galápagos, donde existen alrededor de 444 especies (Grove \& Lavenberg 1997); lo que podría influir directamente en las diferencias que existen en el espectro trófico de la especie, en ambas regiones.

A su vez, se observó un aporte desigual en cuanto a las presas principales que conformaron la dieta de esta especie, ya que el Índice de Importancia Relativa (IIR) reflejó que solamente cuatro presas representaban el $83,4 \%$ de la dieta total. Las dos presas principales estuvieron asociadas a un hábitat mesopelágico resultado que coincide con lo reportado por Watanabe (1958) y Alatorre-Ramírez (2007) para otras zonas del Océano Pacífico Oriental. A su vez se registraron varias especies de peces como presas complementarias, coincidiendo con lo reportado por diversos autores (Watanabe 1958, Alverson 1963, Román-Reyes 2005).

La importancia del calamar gigante Dosidicus gigas en la dieta del atún aleta amarilla, puede deberse a que este depredador se estaría alimentando durante la noche (Schaefer et al. 2007), tiempo en el cual estos calamares realizan sus migraciones verticales desde la zona mesopelágica hacia la superficie para alimentarse (Markaida-Aburto 2001). Generalmente se ha asociado a Dosidicus gigas con zonas de surgencia ricas en nutrientes cercanas al talud continental (Ehrhardt et al. 1986, Markaida-Aburto 2001), características que se presentan en las Islas Galápagos, ya que a pesar de estar en una zona oligotrófica existen puntos de alta productividad primaria localizados principalmente en el lado oeste del archipiélago (Feldman 1985, Palacios et al. 2006), donde ocurre una producción elevada, esporádica e intensa (10-30 mg Chl-a $\left.\mathrm{m}^{-3}\right)$, sucedida por una productividad nominal baja (1-5 $\left.\mathrm{mg} \mathrm{Chl-a} \mathrm{m}^{-3}\right)$ la cual permanece por más tiempo (Banks 2002).
En el caso de Acanthocybium solandri, el IIR reflejó una dieta principalmente ictiófaga, coincidiendo con lo reportado por varios autores para la región tropical del Océano Pacífico y Atlántico (Oxenford et al. 2003, VaskeJúnior et al. 2003, Bocanegra 2007, Frank et al. 2008). A diferencia de estos antecedentes el espectro trófico identificado para Acanthocybium solandri en la RMG resultó ser más estrecho, diferencias que podrían estar asociadas a la disponibilidad de alimento en cada región, tal y como se explicó en el caso de Thunnus albacares, aunque otros aspectos (e.g., artes de captura y esfuerzo pesquero) podrían influir en el espectro dietario encontrado.

Las poblaciones de Acanthocybium solandri presentes en la RMG presentaron una dieta compuesta por presas de diferentes hábitats: epipelágico, bentónico y bento-pelágico, resultados que coinciden con lo reportado por Oxenford et al. (2003) y Frank et al. (2008), quienes mencionan que las poblaciones de Acanthocybium solandri presentes en el Golfo de México y la costa atlántica de Estados Unidos se alimentan principalmente de presas asociadas a bajos rocosos (islotes) y objetos flotantes, entre las cuales destacan varios peces epipelágicos y demersales. De tal forma, las características topográficas y oceanográficas presentes en las Islas Galápagos favorecen la agregación de especies de diferentes ambientes, las cuales podrían ser utilizadas por estos depredadores.

El pez volador Prognichthys tringa, resultó ser la presa principal en la dieta Acanthocybium solandri en la RMG; esta especie generalmente se encuentra asociada con aguas superficiales cercanas a la costa, principalmente en la zona ecuatorial del Océano Pacífico Oriental (Grove \& Lavenberg 1997). Este resultado se ajusta con el área de distribución de Acanthocybium solandri en el ecosistema, ya que muestra una gran dependencia a la zona de la termoclina, por lo que generalmente se distribuye en los primeros $20 \mathrm{~m}$ de la columna de agua (Collette \& Nauen 1983, Bernal et al. 2009, Sepúlveda et al.2011).

Actualmente se desconoce la dinámica espacial y temporal de las principales presas reportadas para estas especies en la RMG; sin embargo estudios enfocados en la dieta de tiburones a nivel del Pacífico ecuatoriano también señalan a Dosidicus gigas como una de las presas más importantes y abundantes dentro del ecosistema pelágico de la región (Polo-Silva 2009, CalleMoran 2010). De tal forma que resultaría lógico que esta presa sea una de las principales en la dieta de Thunnus 
albacares y Acanthocybium solandri, ya que posiblemente sus dietas están relacionadas con la abundancia de presas, las que pueden variar en porcentaje de importancia de acuerdo al lugar y época del año, por lo tanto, al compartir un mismo hábitat las especies desarrollan distintos mecanismos tróficos, enfocados en compartir presas pero con un distinto porcentaje de importancia (Bolnick et al. 2003, Páez-Rosas \& AuriolesGamboa 2010).

Varios estudios han categorizado a los grandes peces pelágicos como especies oportunistas o generalistas, lo que significa que estas especies depredan sobre los recursos disponibles en un lugar y momento dados (Alatorre-Ramírez 2007). El espectro trófico de Thunnus albacares demuestra que poseen una dieta diversa $\left(\mathrm{H}^{\prime}=\right.$ $2,88)$, sin embargo el valor calculado de amplitud trófica lo ubica como un depredador de tipo especialista $(B i=$ $0,17)$, es decir esta especie tienen un amplio espectro alimentario pero depreda principalmente sobre un reducido número de especies presas, cuya magnitud de depredación puede variar en función del tiempo y del espacio, por esto, este tipo de depredadores son considerados como 'especialistas plásticos' (Lowry et al. 1991, Porras-Peters et al. 2008, Calle-Moran 2010, PáezRosas \& Aurioles-Gamboa 2010). De esta forma se evidencia un patrón diferencial en los hábitos alimentarios de estas especies durante la temporada de estudio; Thunnus albacares fue monoespecífico (depredador especialista) enfocado a los calamares, mientras que Acanthocybium solandri fue multiespecífico, a pesar de presentar un espectro trófico más reducido.

Las diferencias en el espectro trófico de ambas especies podrían estar asociadas a su distribución en la columna de agua; se conoce que Acanthocybium solandri generalmente se distribuye por encima de la termoclina (zonas costeras), y limita su desplazamiento a profundidades menores a $20 \mathrm{~m}$ (Collette \& Nauen 1983, Sepúlveda et al. 2011), mientras que Thunnus albacares presenta un mayor intervalo de distribución, llegando a desplazarse hasta profundidades cercanas a los $100 \mathrm{~m}$ (zonas oceánicas) (Schaefer et al. 2007, Bernal et al. 2009).

Otro factor que podría influir en los hábitos alimentarios de estos depredadores sería la densidad de individuos de ambas especies en el ecosistema (Bolnick et al. 2003, Estes et al. 2003). En este sentido se conoce que en ecosistemas expuestos a una sobre pesca la densidad de depredadores tope es relativamente baja (con relación a sus presas), provocando cierta igualdad en el consumo de recursos producto de la poca necesidad de competencia (Bolnick et al. 2003). Mientras que en regiones protegidas de la pesca industrial (Reservas Marinas), la densidad poblacional de la mayoría de depredadores es más alta, lo cual provocaría un incremento en los niveles de competencia trófica (Estes et al. 2003). Este sería el caso de la RMG; por lo cual es esperable que ambas especies estén forzadas a alimentarse en diferentes nichos tróficos, estrategia que la estarían utilizando con la finalidad de repartirse los recursos alimentarios presentes en la zona.

El presente estudio contribuye al conocimiento de la dieta del atún aleta amarilla Thunnus albacares y el guajo Acanthocybium solandri en la región de Galápagos, demostrando ciertas diferencias en el comportamiento trófico de las poblaciones de Thunnus albacares y Acanthocybium solandri presentes en las Islas Galápagos, lo que es interesante, dado que ambas especies aparentemente ocuparían un nicho trófico similar, de tal forma que esta respuesta adaptativa sería el reflejo de una utilización diferencial del hábitat de alimentación por parte de ambas especies, factor que estaría facilitando su coexistencia en la región.

\section{Agradecimientos}

Agradecemos al Fondo para la Conservación de Galápagos (Galápagos Conservation Trust) y a la WWFInternational por el apoyo brindado para la elaboración de proyecto. A la Fundación Charles Darwin y al Parque Nacional Galápagos por la logística y los permisos de investigación. Al Dr. Felipe Galván-Magaña y el personal del Laboratorio de Ecología de Peces del CICIMARMéxico por la accesoria en la fase de identificación de muestras. Al Sector Pesquero Artesanal de Galápagos de la Isla Santa Cruz, en especial al Sr. Carlos Bailón y a su grupo de trabajo, quienes colaboraron en gran medida durante la fase de campo de este proyecto. Al Lic. Cesar Peñaherrera Palma y todo el personal del Área de Ciencias Marinas y Costeras de la Fundación Charles Darwin por su ayuda durante este estudio.

\section{LITERATURA CITADA}

Alatorre-Ramírez V. 2007. Hábitos alimenticios del atún aleta amarilla Thunnus albacares y barrilete Katsuwonus pelamis en cardúmenes mixtos del Océano Pacífico Oriental Tropical. Tesis de Maestría, Instituto Politécnico Nacional, México, $86 \mathrm{pp}$.

Alverson F. 1963. The food of yellowfin and skipjack tunas in the eastern tropical Pacific Ocean. Inter-American Tropical Tuna Commission, Bulletin 7(5): 295-396. 
Arenas P, M Hall \& M García. 1999. Association of fauna with floating objects in the eastern Pacific Ocean. En: Proceedings of the international workshop on the ecology and fisheries for tunas associated with floating objects. IATTC Special Report 11: 285-326.

Au D. 1991. Polyspecific nature of tuna schools: sharks, dolphin and seabirds associates. Fishery Bulletin 89: 343-354.

Banks S. 2002. Ambiente físico. En: Reserva Marina de Galápagos. Línea Base de la Biodiversidad. Fundación Charles Darwin y Servicio Parque Nacional Galápagos, Santa Cruz, Galápagos, 484 pp.

Bernal D, C Sepúlveda, M Musyl \& R Brill. 2009. The ecophysiology of swimming and movement patterns of tunas, billfishes, and large pelagic sharks. In: Fish locomotion-an etho-ecological perspective, pp. 436-483. Science Publishers, Enfield.

Bocanegra N. 2007. Relaciones tróficas de los peces pelágicos asociados a la pesquería del atún en el Océano Pacífico Oriental. Tesis de Doctorado, Instituto Politécnico Nacional, México, 178 pp.

Bolnick D, R Svanbäck, J Fordyce, L Yang, J Davis, C Hulsey \& M Forister. 2003. The ecology of individuals: incidence and implications of individual specialization. American Naturalist 161(1): 1-28.

Caillet G, S Love \& A Ebeling. 1986. Fishes. A field and laboratory manual on their structure identification and natural history, 194 pp. Waveland Press, Illinois.

Calle-Moran M. 2010. Ecología trófica del tiburón zorro pelágico Alopias pelagicus en Santa Rosa de Salinas, Pacífico Ecuatoriano. Tesis de Maestría, Universidad Nacional Autónoma de México, México, 87 pp.

Castrejón M. 2008. El sistema de Co-Manejo Pesquero de la Reserva Marina de Galápagos: Situación actual, retos y perspectivas de cambio. Fundación Charles Darwin. Galápagos.

Clarke M. 1986. A handbook for the identification of cephalopod beaks, 873 pp. Clarendon Press. Oxford.

Clarke K \& R Warwick. 2001. Changes in marine communities: an approach to statistical analysis and interpretation, 172 pp. PRIMER-E, Plymouth.

Clothier C \& J Baxter. 1969. Vertebral characters of some Californian fishes with notes on other Eastern Pacific species, 32 pp. Department of Fish and Game. Marine Resources Operations, Sacramento.

Collette BB \& CE Nauen. 1983. FAO species catalogue, vol 2. Scombrids of the world. An annotated and illustrated catalogue of tunas, mackerels, bonitos, and related species known to date. FAO Fisheries Synopsis 125: 1-137.

Ehrhardt N, A Solis, J Pierre, J Ortiz, P Ulloa, G González \& F Garcia. 1986. Análisis de la biología y condiciones del stock del calamar gigante Dosidicus gigas en el Golfo de California, durante 1980. Ciencia Pesquera 5: 63-76.
Eslava N, L Gonzáles \& D Gaertner. 2003. Asociación de la abundancia y la distribución vertical de atunes y peces de pico en el sureste del Mar Caribe. Revista de Biología Tropical 51(1): 213-219.

Estes JA, ML Riedman, MM Staedler, MT Tinker \& BE Lyon. 2003. Individual variation in prey selection by sea otters: Patterns, causes and implications. Journal of Animal Ecology 72: 144-155.

Ferry L, S Clark \& G Cailliet. 1997. Food habits of spotted sand bass (Paralabrax maculatofasciatus), (Serranidae) from Bahia de Los Angeles, Baja California. Southern California Academy Science 96: 1-21.

Frank JS, ER Hoffmayer, JR Ballard, NM Garber \& AF Garber. 2008. Diet of wahoo, Acanthocybium solandri, from the Northcentral Gulf of Mexico. Proceedings of the 60th Gulf and Caribbean Fisheries Institute, November 59, 2007, Punta Cana, pp. 353-362.

Galván-Magaña F. 1988. Composición y análisis de la dieta del atún aleta amarilla Thunnus albacares en el Pacífico mexicano durante 1984-1985. Tesis de Maestría, Instituto Politécnico Nacional, México, 86 pp.

Galván F, H Nienhuis \& P Klimley. 1989. Seasonal abundance and feeding habits of sharks of the lower Gulf of California, Mexico. California Fish and Game 75: 74-84.

García-Godos I. 2001. Patrones morfológicos del otolito sagitta de algunos peces óseos del mar peruano. Informe, Instituto del Mar, Perú 20: 4-83.

Garth J \& W Stephenson. 1966. Brachyura of the Pacific coast of America. Brachyrhyncha: Portunidae. Allan Hancock Monograph in Marine Biology 1: 1-154.

Grove J \& R Lavenberg. 1997. The fishes of the Galapagos Islands, 863 pp. Stanford University Press, California.

Hastings P, L Findley \& A Van der Heiden. 2010. Fishes of the Gulf of California, 118 pp. Arizona University Press, Tucson.

Hyslop J. 1980. Stomach contents analysis. A review of methods and their application. Journal of Fish Biology 17: 411-429.

Krebs C. 1999. Ecological methodology, 620 pp. Addison Wesley/Longman, Menlo Park.

Lowry MS, BS Stewart, CB Heath, PK Yochem \& JM Francis. 1991. Seasonal and annual variability in the diet of California sea lions (Zalophus californianus) at San Nicolas Island California, 1981-1986. Fishery Bulletin 89: 331-336.

Markaida-Aburto U. 2001. Biología del calamar gigante Dosidicus gigas, Orbigny 1835 (Cephalopoda: Ommastrephidae) en el Golfo de California, México. Tesis de Doctorado, Centro de Investigación Científica y Educación Superior de Ensenada, México, 387 pp. 
Nikolsky G. 1963. The ecology of fishes, $352 \mathrm{pp}$. Academic Press, San Diego.

Olson R \& C Boggs. 1986. Apex predation by yellowfin tuna (Thunnus albacares); independent estimates from gastric evaluation and stomach contents, bionergetics, and cesium concentrations. Canadian Journal of Fisheries and Aquatic Science 43: 1760-1775.

Oxenford H, P Murray \& B Luckhurst. 2003. The biology of guajo (Acanthocybium solandri) in the western central Atlantic. Gulf and Caribbean Research 15: 33-49.

Páez-Rosas D. 2008. Diversificación de dietas en tres colonias de lobo marino de Galápagos, Zalophus wollebaeki, evaluada con análisis de excretas e isótopos estables de $\mathrm{C}$ y N. Tesis de Maestría, Instituto Politécnico Nacional, México, 81 pp.

Páez-Rosas D \& D Aurioles-Gamboa. 2010. Alimentary niche partitioning in the Galapagos sea lion, Zalophus wollebaeki. Marine Biology 157: 2769-2781.

Palacios D. 2002. Factors influencing the island-mass effect of the Galápagos. Geophysical Research Letters 29: 49.

Palacios D, S Bograd, D Foley \& F Schwing. 2006. Oceanographic characteristics of biological hot spots in the North Pacific: A remote sensing perspective. Deep-Sea Research II 53: 250-269.

Pinkas L, M Oliphant \& I Iverson. 1971. Food habits of albacore, bluefin tuna, and bonito in California waters. Fishery Bulletin 152: 1-105.

Polo-Silva C. 2009. Ecología trófica de los tiburones zorro Alopias pelagicus (Nakamura 1935) y Alopias superciliosus (Lowe, 1839) en el Pacífico ecuatoriano. Tesis de Maestría, Instituto Politécnico Nacional, México, 102 pp.

Román-Reyes J. 2005. Análisis del contenido estomacal y la razón de isótopos estables de carbono $(\delta 13 \mathrm{C})$ y nitrógeno $(\delta 15 \mathrm{~N})$ del atún aleta amarilla (Thunnus albacares), delfín manchado (Stenella attenuata) y delfín tornillo (Stenella longirostris) del Océano Pacífico oriental. Tesis de Doctorado, Instituto Politécnico Nacional, México, 143 pp.
Salazar-Alzaz D. 2007. Ecología alimentaria del lobo marino de Galápagos (Zalophus wollebaeki) en el islote Caamaño. Tesis de Licenciatura, Pontificia Universidad Católica, Quito, 122 pp.

Schaefer KM, DW Fuller \& BA Block. 2007. Movements, behavior, and habitat utilization of yellowfin tuna (Thunnus albacares) in the northeastern Pacific Ocean, ascertained through archival tag data. Marine Biology 152: 503-525.

Sepúlveda CA, SA Albers, S Ortega-Garcia, NC Wegner \& D Bernal. 2011. Depth distribution and temperature preferences of wahoo (Acanthocybium solandri) off Baja California Sur, Mexico. Marine Biology 158: 917-926.

Stevens B, D Armstrong \& R Cusiano. 1982. Feeding habits of the Dungeness crab, Cancer magister, as determined by the index of relative importance. Marine Biology 72: 135145 .

Theisen B, W Bowen, W Lanier \& J Baldwin. 2008. High connectivity on a global scale in the pelagic guajo, Acanthocybium solandri (tuna family Scombridae). Molecular Ecology 17: 4233-4247.

Vaske-Júnior T, C Vooren \& R Lessa. 2003. Feeding strategy of yellowfin tuna (Thunnus albacares), and guajo (Acanthocybium solandri) in the Saint Peter and Saint Paul Archipelago, Brazil. Boletim Instituto de Pesca, Brazil 29(1): 173-181.

Watanabe H. 1958. On the difference of the stomach contents of the yellowfin and bigeye tunas from the western ecuatorial Pacific. Bulletin of Japan Sea Regional Fisheries Research Laboratory, Fisheries Agency 7: 72-81.

Wolff C. 1984. Identification and estimation of size from beaks of 18 species of cephalopods from the Pacific Ocean. NOAA Technical Report NMFS 17: 41-50.

Wooton R. 1990. Ecology of teleost fishes, 404 pp. Chapman \& Hall, New York. 